\title{
Social Skills Group for Adults Living with Intellectual Disabilities
}

\section{Fabienne Giuliani and Pierre El Korh}

\begin{abstract}
Psychiatry and Mental Development Department (Community Psychiatry Service, Psychiatry Department, CHUV, Switzerland
\end{abstract}

Corresponding author: Fabienne Giuliani

ĐFabienne.Giuliani@chuv.ch

Psychiatry and Mental Development Department, Community Psychiatry Service, Psychiatry Department, CHUV, Switzerland.

Tel: 41213142185

Citation: Giuliani F, El Korh P. Social Skills Group for Adults Living with Intellectual Disabilities. Clin Psychiatry. 2015, 2:2.

Received: May 02, 2016; Accepted: May 13, 2016; Published: May 18, 2016

\section{Introduction}

Research conducted on Cognitive Behavioral Therapy (CBT) interventions with individuals living with an intellectual disability are constantly increasing and is promising [1-18]. Recently, studies have begun to demonstrate the possibilities of using group behavioral cognitive therapy for this population [19]. The analyses provided by these authors have shown on the one hand that patients have played an active role in the therapy and on the other hand, that the context of the therapeutic group will change patient behavior. At the same time, these studies also reiterate the importance of adapting the discourse to the patient's level of understanding.

The prevalence of mental health disorders especially affective and anxiety disorders, in people with intellectual deficits reaches $40 \%[20,21]$. Mental health disorders can arise from a multitude of factors which influence each other (biological, psychological, sociological and/or environmental). Which is why it is necessary to conduct an initial clinical evaluation in order to gather information on the causes of patient difficulties? Certain authors $[3,22,23]$ have underlined the importance of a behavioral evaluation before any intervention is undertaken.

To guide therapeutic interventions, one must first be able to construct hypotheses on the difficulties the individual encounters, changes to be made for the patient and in his/her environment as well as the procedures to use to facilitate these changes.

\section{Methods}

\section{Evaluation of interpersonal difficulties}

The group addresses the following types of interpersonal difficulties:

1) Social anxiety: Patients may feel very anxious in a certain number of interpersonal situations (for example, at work, in shops or restaurants, on the bus, etc).

2) Lack of social skills: Patients may have difficulty expressing certain needs, ideas or feelings, either in any interpersonal situation or in front of particular individuals. Social skills were evaluated according to Table 1.

3) A poor perception of his or her own skills: Patients may perceive themselves to be socially inept without this being true.

4) A poor perception of the social-interpersonal reality: Patients may erroneously perceive the messages they receive from others and interpret them incorrectly.

5) A combination of these four factors. 
Table 1 psychiatric co-morbidities.

\begin{tabular}{|c|c|}
\hline Co-morbidities & $\begin{array}{c}\text { Patients of the group suffering } \\
\text { from the following disorders: }\end{array}$ \\
\hline Anxiety disorders & $n=10$ \\
\hline Personality disorders & $n=2$ \\
\hline ADHD & $n=1$ \\
ASD & $n=4$ \\
\hline Addictive disorder & $n=1$ \\
\hline
\end{tabular}

\section{Social Skills: Definition}

"Listen to what someone has said to me (understand), select a response and reply (through verbal or nonverbal means), in an appropriate way for a given social situation."

1. Basic social skills:

Take care of one's appearance

Listen

Start a conversation

Introduce oneself

Answer the telephone

Say thank you...

2. Advanced social skills:

Apologize

Give and follow instructions

Request and offer help

Share activities, materials...

Prepare oneself for a conversation, an interview

3. Recognition and expression of emotions

Identify one's own emotions

Identify other people's emotions

Express feelings, emotions

Respond to teasing

Recognize and use humor, irony

Accept failure...

4. Self-affirmation

Know how to formulate a refusal

Know how to accept a refusal

Know how to make a request

Know how to respond to a request

Know how to make a criticism

Know how to respond to a criticism

Know to how solve a problem...

5. Friendly and affective relationships

How to have friends
How to undertake a romantic relationship.

The following are the four main points to note during the initial evaluation regarding interpersonal difficulties

1) What are the difficulties, among those I have just written, that the patient encounters?

2) In which situations do these difficulties appear? The term "situations" is taken here in the broad sense, including physical and interpersonal aspects, verbal and nonverbal actions and reactions of others. In other words, everything in the patient's environment that can call forth or reinforce the patient's behaviors, emotions and cognitions,

3) What are the behavioral, emotional, and cognitive "chains" as well as what situations lead the patient to manifest problematic behaviors?

4) What are the behaviors, emotions or cognitions that would be better for the patient to learn and manifest in order to resolve these difficulties?

There is no ideal method to access these data. Many different ones should be used. The tools usually used are: questionnaires, self-observations, peer evaluation, role playing and interviews.

Practice of social skills groups

Make-up of the groups

Our patients $(n=10)$ are adults between the ages of 21 and 57 (average of $31.8 \pm 14$ ) who suffer from slight intellectual disabilities (F70 [315.8]) the degree of which has been evaluated with the WAIS-IV [24] accompanied by a psychiatric co-morbidity described in Table 1.

Inclusion criteria are:

(1) Participants fit the diagnostic criteria for mental retardation according to the DSM-IV;

(2) Participants have clinically significant symptoms of anxiety;

(3)They received a score between 41 - 50 of the Global Assessment of Functioning scale from the DSM-IV [25];

(4) Participants were between the ages of 18 and 65 years.

Exclusion criteria are:

(1) Participants suffering from post-traumatic stress syndrome, or disorders related to substance abuse;

(2) Severe psychiatric co-morbidities which prevent their ability from interacting in a group.

We obtained consent from all participants as well as from their legal representatives for group participation and the publication of our results.

The group, which is semi-open, is comprised of 10 monthly 90-minute sessions per year, with a pause/snack of 15 minutes. All sessions are recorded. Referral to the group is decided during a seminar of our unit, given the fact that all patients are being treated in individual therapy sessions by the members of our team. The individual follow-up goes over what the group has worked on in order to transfer and generalize knowledge acquired within the group. The group is meant for people living with an intellectual disabilities and a lack of social skills. To begin with, we specified the interpersonal difficulties in the way that 
we described them previously using the scales we will present hereafter. The facilitators of the group are the first and second authors. The authors are experienced and hold a specialist's degree in Cognitive Behavioral Therapy.

We developed own approach inspiration by CBT $[26,27]$ and we are going to publish the approach in the form of a therapeutic manual meant for patients living with intellectual impairments. We adapted the theoretical support materials to the particularities of understanding and language of the participants. For example, we offer here an example of one of the materials meant for patients (Figure 1).

\section{Sessions are organized in the following manner}

Sessions begin via order of the day, meaning a round table discussion of the problematic subjects that the patient may have experienced and wants to tackle in the group. We note all subjects on a board and we vote upon which subjects the group wants to work on. The other subjects are listed on a monitoring sheet so there is a record of all subjects brought up as well as the individuals who brought them to the group.

\section{Session Program}

\section{Session 1}

We cover what it means to participate in this group: the notion of social skills, the notion of self-esteem, the feeling of selfsufficiency, the notion of stressors and the notion of learning with one's peers. We insist upon the aspects of confidentiality in the group, of written consent for video recording of the sessions and their usefulness.

During this session, we discuss the functional analysis of the situations to be discussed through the vicious circle described by Cungi [28], which enables us to discuss cognitions, emotions, behaviors and consequences (Figure 2).

After completing the functional analysis, we go around the table and list participant experiences of this particular experience. We conduct role plays for this situation. Finally, we finish by going around the table and giving compliments.

At the end of the session, we evaluate the session using a questionnaire which specifies participant anxiety on a scale of 0 to 10 before and after the group; the usefulness and enjoyment of the group activity as well as the quality of the animation (energy and flexibility) are evaluated using a Likert scale.

\section{Session 2 - 10}

The following nine sessions occur in the same manner as the first. For the situations brought to discussion, the following techniques are used according to their appropriateness:

Rules for self-affirmation, relaxation (through square breathing); formulation of a request or a refusal; formulating or receiving a criticism; managing interpersonal conflicts; working on problem-solving techniques; knowing how to make and receive compliments; knowing how to manage and express negative emotions linked to grieving.

\section{Role playing}

Starting from the situation brought up, role playing games are proposed and undertaken by the participants. If one of the participants is having trouble, we offer help by whispering responses and we repeat the situation until he can say it himself. At the end of every role play, we always validate the participant. We use an evaluation table to evaluate participant behaviors on the verbal and nonverbal level (Table 2).

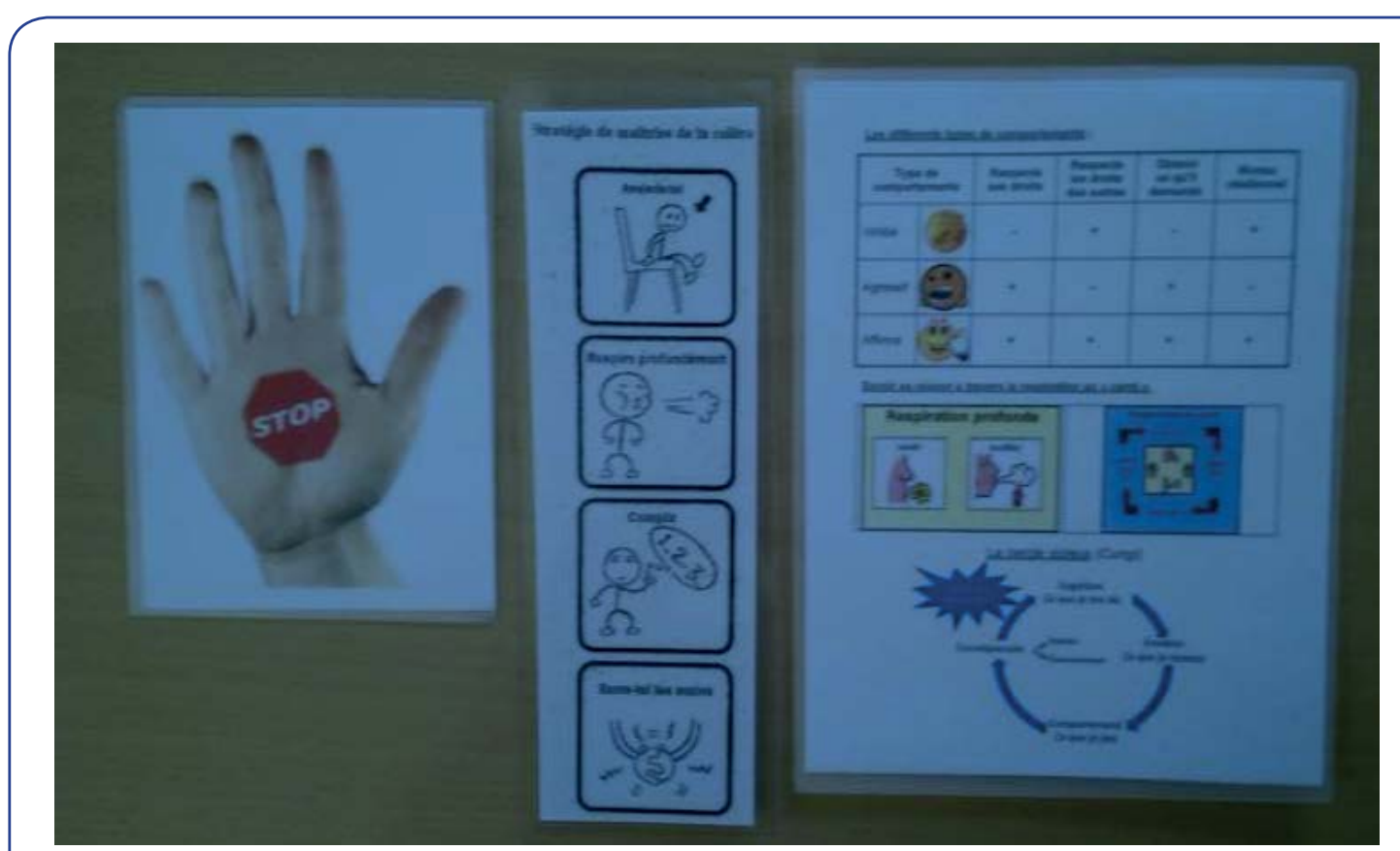

Figure 1 Example material given to patients. To the left, "stop" pictogram to use in social interactions. In the middle, pictogram to control one's anger. To the right, various self-affirmation, breathing, vicious circle (Cungi) techniques. 


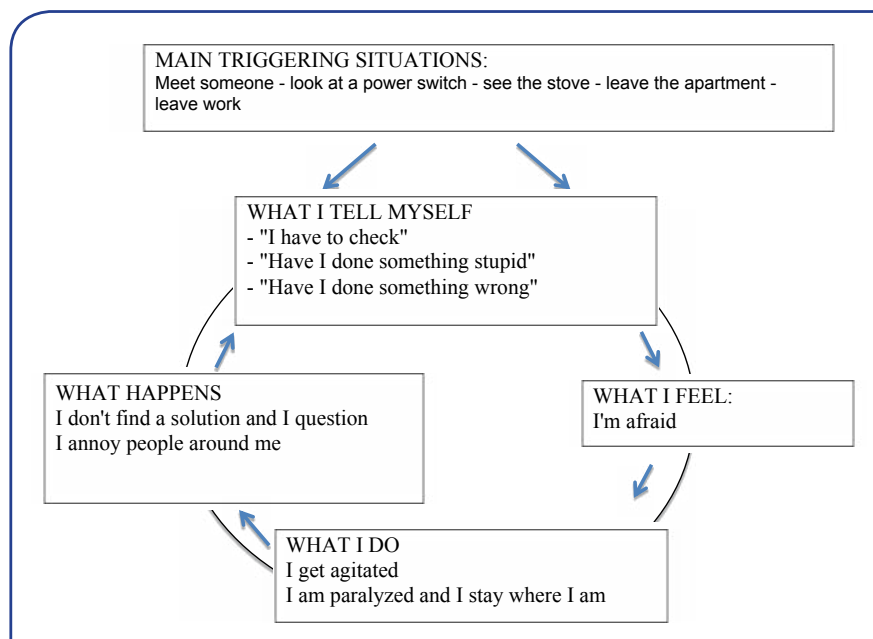

Figure 2 Example of a patient's vicious circle occurring during a group session.

\section{Learning by one's peers}

We focus group work on synergy and the notion of reinforcement by the experiences between participants. Participants allow themselves more easily to accept progress and we work on selfreinforcement which is then amplified through peer feedback. Thanks to a sharing of skills, we estimate that we can work on skill transfer, comparing representations and solidarity between participants. We actively work on the concepts of confidence, identity, belonging and competence.

Finally, two of the decisive factors for the success of this group therapy are:

(1) The participants' motivation to overcome their difficulties,

(2) Their capacities for understanding and conceptualization.

\section{Measurements taken}

We conducted a number of psychopathological evaluations. Measurements were taken at T0, T1 and T2, and were the following:

Beck Anxiety Inventory:

- 13-item Beck Depression Inventory

- Rosenberg Self-Esteem Scale

- The STAI (French version)

We also evaluated participant satisfaction in the group in the following way:

- Anxiety state before/after the group

- Usefulness of the group

- Was participating in the group enjoyable.

- Were the facilitators energetic?

- Were the facilitators flexible in group interactions?

Repeated measures ANOVA (with Stat view 4.5) were used in order to compare the results obtained between T0, T1 and T2, at first on the different scales but also on each group participation. Fisher's post-hoc and other simple correlation comparisons were performed.

\section{Results}

The results obtained show that during the baseline phase (TO), the patients did not suffer from depression, but other questionnaires revealed anxiety disorders in every patient accompanied by serious physiological symptoms evaluated using Beck's Anxiety Inventory. As Figure $\mathbf{3}$ indicates, our therapeutic intervention (T1), as shown by the repeated measures ANOVA on the different phases (T0, T1 and T2), significantly reduced the anxiety disorders $F(3,20=70.206, p<0.0001)$ (Figure 3).

Thanks to the last evaluation, conducted three months after the treatment (T2 = follow-up phase), we were able to report that the improvement obtained in T1 was not a placebo effect, meaning that it was not linked to the patient's positive expectations or a spontaneous positive evolution. It was the stabilization of the results obtained between the intervention and the nonintervention which enabled us to prove the effectiveness of the group therapy work.

For continuous short-interval measurements, we have the responses obtained at the end of each group session. The Spearman rank correlation of the entire data reveal a correlation between the anxiety state (Figure 4 a) before and after the group ( $r h o=.694, d f=139, p<0.0001$ ) as well as the usefulness (Figure $4 b$ ) of the group (rho $=.243, d f=139, p=.0041$ ) (Figure 4).

\section{Discussion}

The data we obtained in the group therapy are in line with the data gathered by Dagnan and et al. $[19,29]$ which suggest that there is a deep engagement on behalf of the participants in the process of patient change. This high level of participation essentially comes from the fact that we focused the group's efforts on synergy and the idea of the reinforcement of experience between participants. The participants allowed each other to more readily accept progress coming from peers who are more similar to them than professionals. Thanks to a sharing of skills, we were able to transfer skills, a comparison of representations and support between participants. These bigger changes linked to group therapy were discussed in a book [30].

As Dagnan and et al. [4], highlighted, there is a considerable potential in terms of the interactions between participants living with a mental handicap in CBT group therapy. The literature on CBT interventions for individuals living with a mental handicap is still at a stage in which descriptive process research is enriching our understanding of group therapy for this particular population. The fact that patients were able to benefit at the same time from an individual follow-up and the social skills group potentiated the effectiveness of the therapy. Despite this, in the future it would be useful to make a distinction between a group who undergo only an individual follow-up and another with a group follow-up on the theme of social skills. Furthermore, the number of individuals participating in the study was small. A larger sample size would have made it possible to validate our results further and make a larger generalization. More studies should be conducted with the same protocol but on a larger number of patients in order to verify the reproducibility of our results. The description of the tools used will be useful for future studies; for example, to 
Table 2 protocol for role playing session.

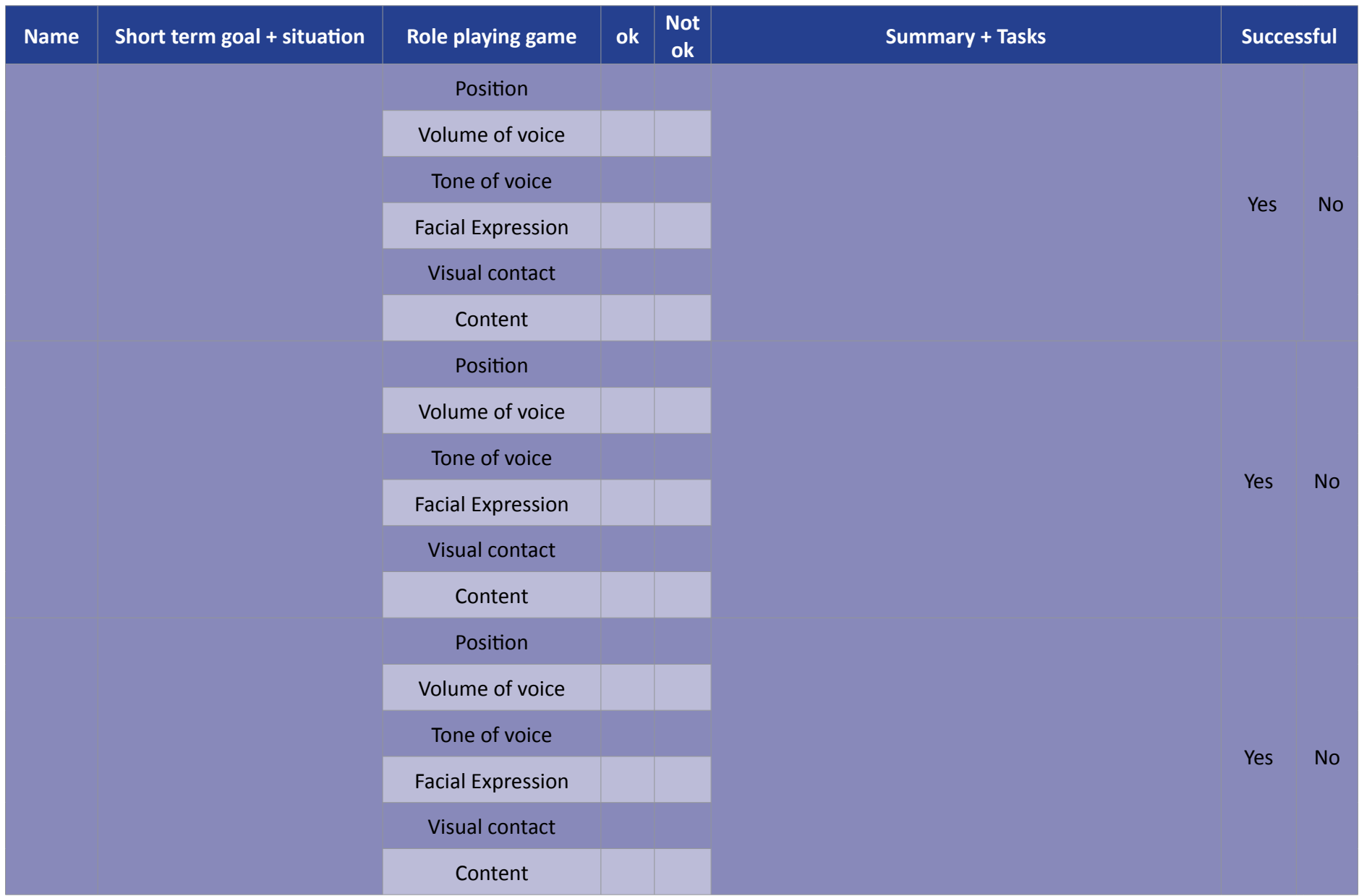

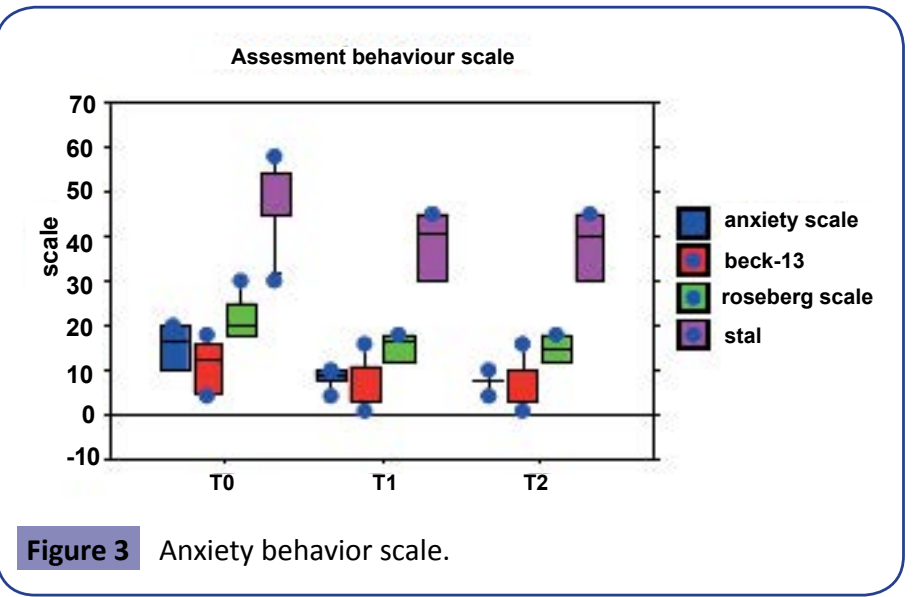

compare animation styles and facilitator flexibility with respect to individuals living with a mental handicap and suffering from depression or schizophrenia, or even to explore the socratic questioning in more detail [31]. Similar to the studies by Willner $[12,16]$ our study shows that the work on social skills makes it possible for patients to reduce their anxiety and acquire selfcontrol over their behaviors and especially over their challenging behaviors. There appeared an improvement in affirmation despite the difficulties in comprehension and language-based expression. This was already found in previous research.

The therapeutic alliance with group participants, their availability and their engagement in the process of cognitive behavioral therapy work also made it possible to reduce their most significant anxiety and which was restricting their autonomy, their self-image and their self-control.

The psycho-educational approach also helped the participants understand the phenomena triggering their difficulty and the related consequences.

Two major conclusions can be drawn from our study. First, it shows that individuals living with intellectual disabilities can improve their social skills in a group setting. Thanks to this work, the patients reduced their anxiety (expressed, by example, by hypervigilance or nervousness) and behavioral problems (asking the same question over and over, for example, becoming aggressive, insulting or even leaving work after an altercation). Secondly, it confirms that personnel can offer CBT interventions that are effective and adapted to this population provided that they are trained [32-38].

The care personnel is an unused resource which can be used 
Evaluation de l'anxiété avant et après le groupe

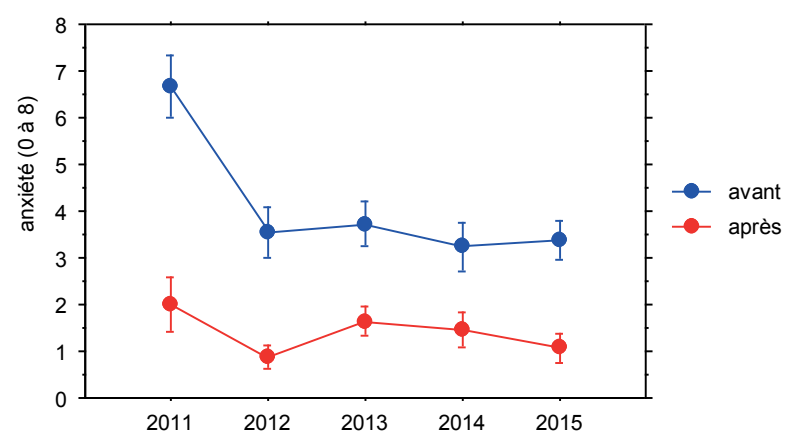

Evaluation du groupe par les participants

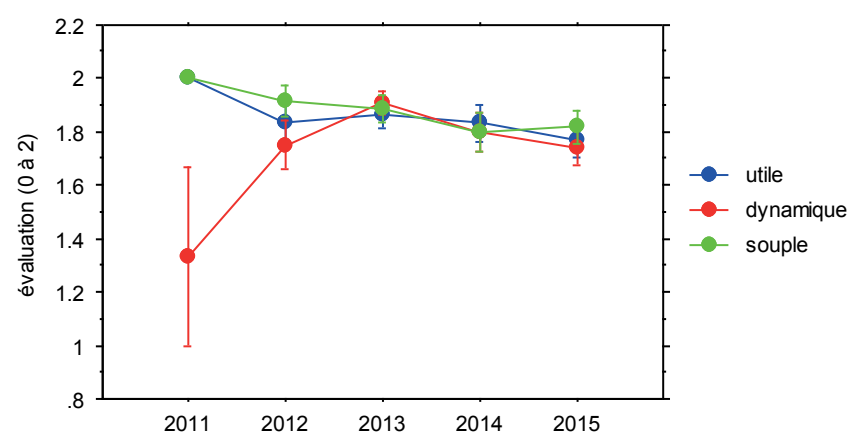

Figure 4 Correlation anxiety scale. to increase the availability of CBT interventions for this often overlooked population. Recognizing the mental health issues of this vulnerable population and increasing the number of psychiatrists specialized in this area [39] are major challenges for the future.

\section{Acknowledgement}

We are grateful for the participants who gave us their trust, as well as everyone in our team who worked together in this therapeutic approach. 


\section{References}

1 Ali A (2015) Behavioural and cognitive-behavioural interventions for outwardly - directed aggressive behaviour in people with intellectual disabilities. Cochrane Database Syst Rev 4: CD003406.

2 Bruce M (2010) Does training improve understanding of core concepts in cognitive behaviour therapy by people with intellectual disabilities? A randomized experiment. Br J Clin Psychol 49: 1-13.

3 Curran JP (1980) The socially inadequate patient: incidence rate, demographic and clinical features, and hospital and posthospital functioning. J Consult Clin Psychol 48: 375-82.

4 Dagnan D (2015) Using Questions in Cognitive Therapy with People with Intellectual Disabilities. Behav Cogn Psychother pp: 1-5.

5 Hassiotis A (2011) Cognitive behaviour therapy (CBT) for anxiety and depression in adults with mild intellectual disabilities (ID): a pilot randomised controlled tria 2: 95.

6 Kellett S, Matuozzo H, Kotecha C (2015) Effectiveness of cognitivebehaviour therapy for hoarding disorder in people with mild intellectual disabilities. Res Dev Disabil 47: 385-92.

7 Lancioni GE (2009) An overview of behavioral strategies for reducing hand-related stereotypies of persons with severe to profound intellectual and multiple disabilities: 1995-2007. Res Dev Disabil 30 20-43.

8 Nicoll M, Beail N, Saxon D (2013) Cognitive behavioural treatment for anger in adults with intellectual disabilities: a systematic review and meta-analysis. J Appl Res Intellect Disabil 26: 47-62.

9 Pert C (2013) Cognitive behavioural therapy from the perspective of clients with mild intellectual disabilities: a qualitative investigation of process issues. J Intellect Disabil Res 57: 359-69.

10 Reddy V (2015) Inpatient group therapeutic interventions for patients with intellectual disabilities. J Intellect Disabil 19: 51-7.

11 Rose J (2013) A preliminary investigation into the influence of therapist experience on the outcome of individual anger interventions for people with intellectual disabilities. Behav Cogn Psychother 41: 470-478.

12 Stimpson A (2013) The experiences of staff taking on the role of lay therapist in a group-based cognitive behavioural therapy anger management intervention for people with intellectual disabilities. J Appl Res Intellect Disabil 26: 63-70.

13 Tomasulo DJ (2014) Positive group psychotherapy modified for adults with intellectual disabilities. J Intellect Disabil 18: 337-50.

14 VereenoogheL, Langdon PE (2013) Psychological therapies for people with intellectual disabilities: a systematic review and meta-analysis. Res Dev Disabil 34: 4085-102.

15 Willner P, Jahoda ALP (2013) Management of anger in people with intellectual disabilities. , in Theory, Application, and Outcome Fernandez E, Editor, Oxford University Press: Oxford.

16 Willner P (2013) Group-based cognitive-behavioural anger management for people with mild to moderate intellectual disabilities: cluster randomised controlled trial. Br J Psychiatry 203: 288-96.

17 Wong DF, Poon A, Kwok YC (2011) The maintenance effect of cognitive-behavioural treatment groups for the Chinese parents of children with intellectual disabilities in Melbourne, Australia: à 6-month follow-up study. J Intellect Disabil Res 55: 1043-53.

18 Zijlmans LJ (2011) Training emotional intelligence related to treatment skills of staff working with clients with intellectual disabilities and challenging behaviour. J Intellect Disabil Res 55: 219-30.
19 Jahoda A (2009) The balance of power in therapeutic interactions with individuals who have intellectual disabilities. $\mathrm{Br} J$ Clin Psychol 48: 63-77.

20 Rojahn J (2003) The Aberrant Behavior Checklist and the Behavior Problems Inventory: convergent and divergent validity. Res Dev Disabil 24: 391-404.

21 Rojahn JMJT (1996) Psychopathology in mental retardation, in Manual of diagnosis and professional practice in mental retardation, JWJaJA Mujick, Editor, APA: Washington p: 147-156.

22 Boisvert JM (1987) The developement of social competence : a practical guide behavior modification Review p: 17(1).

23 Kazdin AE (1979) Unobtrusive measures in behavioral assessment. J Appl Behav Anal 12: 713-24.

24 Wechsler D, WAIS-IV (2011) Wechsler Intelligence Scale for adults. Publishing Center of Applied Psychology ECPA - Edition Paris.

25 DSM-IV ed (2000) DSM-IV-TR : diagnostic and statistical manual of mental disorders. 4th ed text revision ed. 2000, American Psychiatric Association: Washington, DC. XXXVII, 943.

26 Cungi C (2001) Cognitive therapy : manual therapist. 2e edition ed, Rumilly: Afforthecc.

27 Cungi C (2001) Assertiveness : Therapist manual. Rumilly: Afforthecc.

28 Cungi CSsaPR (1996) Being assertive. Retz ed, Paris.

29 Hargie O, Dickson D (2004) Skilled Interpersonal Communication: research, theory and practice Routledge Hove, East Sussex.

30 James IA, Morse R, Howarth A (2010) The science and art of asking questions in cognitive therapy. Behav Cogn Psychother 38: 83-93.

31 Giuliani F, El Korh P (2014) Psychotherapy of people with Asperger syndrome around the caetextia. Swiss Archives of Neurology and Psychiatry 165 : 298-305.

32 Giuliani F, El Korh P (2015) Case study: the contribution of the neurosciences in the treatment of the anxiety disorders at two people living with autism spectrum disorders and intellectual disabilities. RFCCC 10: 8-22.

33 Giuliani F, El Korh P (2016) Is Storytelling Therapy Useful for Children with Autism Spectrum Disorders and Severe Mental Retardation? Advanced Techniques in Biology \& Medicine 4: 166-201.

34 Giuliani F, El Korh P (2016) Adult with Autistic Spectrum Disorders: Specialized Treatment. Advanced Techniques in Biology \& Medicine 4: $164-8$

35 Giuliani F, Perrenoud V, Favrod J (2014) Using eye-tracking as support for the TEACCH program and two teenagers with autism-spectrum disorders. Francophone journal Behavioral and Cognitive Clinic XIX: 39-56.

36 McGillivray JA, McCabe MP, Kershaw MM (2008) Depression in people with intellectual disability: an evaluation of a staff-administered treatment program. Res Dev Disabil 29: 524-36.

37 Willner P, Tomlinson S (2007) Generalization of anger-coping skills from day-service to residential settings. J Appl Res Intellect Disabil 20: 553-62.

38 Health Do (2007) Choices in Mental Health: Improving Access to Psychological Therapies, DoHCSIPDo Health, Editor.

39 Grasset $F$ (2008) Mental retardation and psychiatry. Rev Med issue 4: 1976-8, 1980-1. 